Eastern Illinois University

The Keep

Faculty Research and Creative Activity

Kinesiology \& Sports Studies

May 2013

\title{
Supra-physiological doses of testosterone affect membrane oxidation of human neutrophils monitored by the fluorescent probe C11-BODIPY581/591
}

Tacito Pessoa de Souza Jr

Paraná Federal University

Andre K. Yamada

São Carlos Federal University

Roberto Simao

Federal University of Rio de Janeiro

Tatiana G. Polotow

Institute of Physical Activity and Sports Sciences (ICAFE), Universidade Cruzeiro do Sul

Rui Curi

University of Sao Paulo

See next page for additional authors

Follow this and additional works at: http://thekeep.eiu.edu/kss_fac

Part of the Kinesiology Commons

\section{Recommended Citation}

de Souza, Tacito Pessoa Jr; Yamada, Andre K.; Simao, Roberto; Polotow, Tatiana G.; Curi, Rui; Pope, Zachary; Willardson, Jeffrey; and Barros, Marcelo P., "Supra-physiological doses of testosterone affect membrane oxidation of human neutrophils monitored by the fluorescent probe C11-BODIPY581/591" (2013). Faculty Research and Creative Activity. 29.

http://thekeep.eiu.edu/kss_fac/29

This Article is brought to you for free and open access by the Kinesiology \& Sports Studies at The Keep. It has been accepted for inclusion in Faculty Research and Creative Activity by an authorized administrator of The Keep. For more information, please contact tabruns@eiu.edu. 


\section{Authors}

Tacito Pessoa de Souza Jr, Andre K. Yamada, Roberto Simao, Tatiana G. Polotow, Rui Curi, Zachary Pope, Jeffrey Willardson, and Marcelo P. Barros 


\title{
Supra-physiological doses of testosterone affect membrane oxidation of human neutrophils monitored by the fluorescent probe $\mathrm{C}_{11}$-BODIPY581/591
}

Tácito Pessoa de SouzaJunior, André K. Yamada, Roberto Simão, Tatiana G. Po lotow, Rui Curi, Zachary Pope, Jeffrey M. Willardson and Marcelo P. Barros

\begin{abstract}
The purpose of this study was to determine the effects of supra-physiological doses of testosterone (TES) on membrane oxidation of activated human neutrophils in vitro using an innovative and sensitive technique: the realtime detection with the fluorescence probe C11-BODIPY581/591.

Methodological controls were performed with the lipid-soluble and powerful antioxidant astaxanthin at different neutrophil density cultures. Neutrophils from nine healthy young men $(23.4 \pm 2.5$ years, $174.4 \pm 7.0 \mathrm{~cm}$ height, and $78.3 \pm 7.0 \mathrm{~kg}$ weight) were isolated and treated with 0.1 or $10 \mu \mathrm{M}$ TES for 24 $\mathrm{h}$ and subsequently labeled with the free radical-sensitive probe C11BODIPY581/591 for monitoring membrane oxidation after neutrophil activation with phorbol-12-myristate-13-acetate (PMA). First-order exponential decay kinetic indicated that both 0.1 and $10 \mu \mathrm{M}$ TES severely increased baseline membrane oxidation in non-activated human neutrophils (compared to control). However, similar kinetics of membrane oxidation were observed in control and $0.1 \mu \mathrm{M}$ TES-treated neutrophils after PMA activation, whereas chemical activation did not alter the baseline higher rates of membrane oxidation in $10 \mu \mathrm{M}$ TES-treated neutrophils. The data presented here support the hypothesis that TES exerts distinct effects on the membrane oxidation of human neutrophils, depending on its dose (here, 102 to 104-fold higher than physiological levels in men) and on PMA activation of the oxidative burst. Furthermore, this paper also presents an innovative application of the free radical-sensitive probe C11-BODIPY581/591 for monitoring (auto-induced) membrane oxidation as an important parameter of viability and, thus, responsiveness of immune cells in inflammatory processes.
\end{abstract}

Keywords: Lipoperoxidation, Steroid, Astaxanthin, Antioxidant, Immune, Oxidative stress

\section{Abbreviations}

AAS

Androgen-anabolic steroids

AST

Astaxanthin

$C_{11-B O D I P Y 581 / 591}$ 
4,4-Difluoro-5-(4-phenyl-1,3-butadienyl)-4-bora-3a,4a-diaza-s-indacene-3undecanoic acid

\section{DMSO}

Dimethylsulfoxide

HEPES

4-(2-hydroxyethyl)-1-piperazineethanesulfonic acid

PMA

Phorbol-12-myristate-13-acetate

ROS/RNS

Reactive oxygen/nitrogen species

TBARS

Thiobarbituric acid reactive substances

TES

Testosterone

VSMCs

Vascular smooth muscle cells

\section{Introduction}

Anabolic-androgenic steroids (AAS) have been long used in the treatment of chronic debilitating illnesses, trauma, burns, surgery, and radiation therapy (Shehzad et al. 2001). The desire for improved body aesthetics, physical function, and sport performance has also fueled anabolic drug abuse over the past 50 years (Hoffman et al. 2009). Since aging is regularly associated with a gradual decline in circulating testosterone concentrations and decreased musculature in men, AAS replacement therapies have been currently suggested to circumvent the age-related morbidity and cognitive impairment in men (Dillon et al.2010). Accordingly, supra-physiologic ( $50 \mathrm{nM}=50$-fold normal) dosing of testosterone for eight continuous weeks has been shown protective to muscle mass and fatigue resistance in orchiectomized mice compared to sham operated animals (Axell et al. 2006). The protective action of testosterone on skeletal muscle and cognitive capacities may in part be due to its anti-inflammatory actions or through direct modulation of anti-catabolic pathways (Thompson et al. 2006; Liva and Voskuhl 2001; Malkin et al. 2004). On the other hand, concerns about the potential adverse effects of higher doses of testosterone have encouraged the development of strategies to define a possible "pharmacological window" for beneficial AAS effects in men.

Regarding inflammatory responses, increased levels of pro-inflammatory cytokines (e.g., IL-8, TNF $\alpha$ ) stimulates the migration of circulating neutrophils to the inflammatory site, followed by activation of the membrane enzyme NADPH oxidase and massive production of reactive oxygen/nitrogen species (ROS/RNS) (Dong et al. 2011; Krüeger et al. 2009; Zembron-Lacny et al. 2010). Although the oxidative burst of neutrophils represents a key-event in eliciting efficient immune responses, the massive production of ROS/RNS can also negatively: (a) affect the structural integrity of the neutrophil membrane; (b) limit the microbicidal and phagocytic activities of neutrophils due to oxidative modification of membrane proteins; (c) 
induce apoptosis prematurely leading to cell degradation; (d) shorten the half-life of active neutrophils in inflammatory sites; and, as a general effect; (e) compromise general immune responsiveness (Ottonello et al.2011). Lipid/membrane oxidation is notoriously recognized as a harmful event related to cell dysfunction and, thus, possibly related here to loss of neutrophil viability during inflammatory processes. Thus, by strategically pre-attaching the free radical-sensitive fluorescent probe $\mathrm{C}_{11}$ BODIPY581/591 (Drummen et al.2002) to neutrophil membranes, we aim here to investigate the effects of supra-physiological doses $(0.1$ and $10 \mu \mathrm{M})$ of testosterone on the membrane oxidation kinetics of activated human neutrophils in vitro, as an important marker of immune efficiency. Furthermore, this paper also presents an innovative application of $\mathrm{C}_{11}$-BODIPY581/591 for monitoring the progression of the auto-induced membrane oxidation in immune cells. Proper methodological controls were performed with the powerful lipid-soluble antioxidant astaxanthin (Barros et al. 2001; Naguib 2000) in order to check the susceptibility of neutrophil membranes to ROS/RNS-especially peroxyl and alkoxyl radicals, main propagating agents of lipid oxidation-and the effects of cell density to the fluorescence signal.

\section{Materials and methods}

\section{Chemicals}

Highly purified chemicals for usual solutions, including specific buffers, were purchased from Labsynth (Diadema, SP, Brazil). Other chemicals like Histopaque1077, dimethyl sulfoxide (DMSO), Triton X-100, astaxanthin, and phorbol-12myristate 13-acetate (PMA) were obtained from Sigma-Aldrich Chemical Co (St Louis, MO, USA). The fluorescent probe 4,4-difluoro-5-(4-phenyl-1,3-butadienyl)-4bora-3a,4a-diaza-s-indacene-3-undecanoic acid (C11-BODIPY581/591) was purchased from Molecular Probes (Ontario, Canada).

\section{Subjects}

Nine healthy, untrained young men (undergraduate students from University Metropolitan of Santos, Brazil) volunteered to participate in this research. Subject (mean \pm SD) age, height, and body mass were $(23.4 \pm 2.5)$ years $(174.4 \pm 7.0) \mathrm{cm}$, and $(78.3 \pm 7.0) \mathrm{kg}$, respectively. Blood collecting protocol and all other experimental procedures were approved by the Ethics Committee in Research of University Cruzeiro do Sul (Ethical Protocol CEP-002-2011), which conforms to the Standards for Research Using Human Subjects, Resolution 196/96 of the USA National Health Council of 10/10/1996.

\section{Human neutrophils in culture}

Blood samples (10 mL) were drawn from the forearm cubital vein of volunteers using EDTA-containing Vacutainer ${ }^{\circledR}$ kits. Heparinized tubes were avoided, since eventual iron contamination could accelerate oxidative degradation of samples (Freitas et al. 2008). Blood samples were diluted in the same volume of phosphate 
buffered saline solution (0.137 $\mathrm{M} \mathrm{NaCl}, 2.7 \mathrm{mM} \mathrm{KCl}, 8.0 \mathrm{mM} \mathrm{Na}_{2} \mathrm{HPO}_{4}, \mathrm{pH} 7.4$ ) and neutrophils were separated using Histopaque 1077 according to the manufacturer's instructions (Sigma-Aldrich, MO, USA). The plasma and intermediary layer were removed and both neutrophils and erythrocytes were collected from sediment. Erythrocytes were lysed with a hemolysis solution $\left(150 \mathrm{mM} \mathrm{NH}_{4} \mathrm{Cl}, 10 \mathrm{mM} \mathrm{NaHCO}\right.$, $0.1 \mathrm{mM}$ EDTA, pH 7.4) and immediately centrifuged for $10 \mathrm{~min}\left(600 \mathrm{~g}\right.$ at $\left.4{ }^{\circ} \mathrm{C}\right)$. This procedure was repeated twice for full red blood cell lyse. Thereafter, neutrophils were washed with PBS followed by centrifugation for $10 \mathrm{~min}\left(600 \mathrm{~g}\right.$ at $\left.4^{\circ} \mathrm{C}\right)$. After centrifugation, isolated neutrophils were maintained in RPMI-1640 medium supplemented with $2 \mathrm{mM}$ glutamine, $20 \mathrm{mM}$ HEPES, $10 \%$ fetal calf serum, $10 \mathrm{U} / \mathrm{mL}$ penicillin, and $10 \mathrm{mg} / \mathrm{mL}$ streptomycin. The number of viable cells (>95\%) was determined in a Neubauer chamber under an optical microscope by Trypan blue exclusion (Marin et al. 2010). Alternatively, a second set of human neutrophils were isolated from blood samples by including a pre-cleaning treatment with Dextran 500. Blood samples ( $5 \mathrm{~mL}$ ) were mixed with $6.7 \mathrm{~mL}$ of $6 \%$ non-pyrogenic Dextran $500(\mathrm{w} / \mathrm{v})$ in sterile $0.9 \% \mathrm{NaCl}$ solution and the upper cell layer was transferred to a 50-mL polypropylene tube for a brief centrifugation $(740 \mathrm{~g}$ for $10 \mathrm{~min}$, room temperature). Afterwards, supernatant was discarded and the total cell pellet was resuspended in sterile $0.9 \% \mathrm{NaCl}$ solution. Next, the diluted white blood cell suspension was carefully applied on top of $7 \mathrm{~mL}$ of Histopaque 1077 (in a 50-mL polypropylene tube), as previously described. All materials used for blood collection (syringes, needles, bottles) were disposable and handled by health/medical professionals of the ICAFE/Universidade Cruzeiro do Sul to prevent any potential physical complication.

\section{Testosterone treatment}

Neutrophils $\left(2 \times 10^{6} \mathrm{cell} / \mathrm{mL}\right)$, in a RPMI-1640 medium, were treated with 0.1 and $10 \mu \mathrm{M}$ testosterone $(1.0 \mathrm{mg} / \mathrm{mL}$ in 1,2-dimethoxyethane, T5411-1ML, SigmaAldrich), and incubated in a humidified atmosphere of $5 \% \mathrm{CO}_{2}$ at $37{ }^{\circ} \mathrm{C}$ for $24 \mathrm{~h}$, in accordance with preliminary studies (Marin et al. 2010). Control cultures (treated with same volumes of the drug vehicle, 1,2-dimethoxyethane or DMSO) were simultaneously incubated under the same experimental conditions. Neither 1,2dimethoxyethane nor DMSO (vehicles for TES administration) showed significant toxic effects to isolated neutrophils at concentrations applied here (lower than $2 \%$ of total medium volumes).

\section{Control assays with astaxanthin (AST)}

In order to examine the susceptibility of neutrophil membranes to (auto-induced) oxidation, human neutrophils in culture were pre-loaded with 5 or $10 \mu \mathrm{M}$ astaxanthin (AST), a powerful antioxidant carotenoid recognized by its highly specific scavenging activity against the main propagating agents of lipid oxidation processes: peroxyl and alkoxyl radicals (ROO and RO; respectively). Thus, it is expected that AST could intercept both ROO - and RO radicals and prevent neutrophil membrane from oxidation in active neutrophils. Noteworthy, AST does 
not affect sensitivity or the electronic configuration/properties (thus, fluorescence)

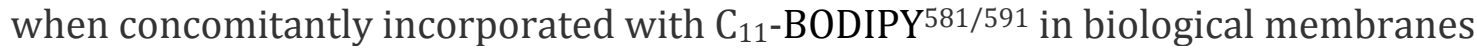
(Naguib 2000). The effects of cell density (5 × 105, $1 \times 10^{6}$, and $2 \times 10^{6}$ cell/well) were also checked in control experiments.

\section{C11-BODIPY581/591 loading in membranes}

Cultured neutrophils were concentrated by centrifugation $(740 \mathrm{~g}$ for $10 \mathrm{~min}$ at room temperature), resuspended in $\mathrm{PBS}\left(0.137 \mathrm{M} \mathrm{NaCl}, 2.7 \mathrm{mM} \mathrm{KCl}, 8.0 \mathrm{mM} \mathrm{Na}_{2} \mathrm{HPO}_{4}, \mathrm{pH}\right.$ 7.4), and seeded in 96-well fluorescence microplates $\left(1 \times 10^{5} /\right.$ well). Subsequently, neutrophils were incubated with $3.2 \mu \mathrm{M} \mathrm{C}_{11}$-BODIPY581/591 (in DMSO), in the dark, at $37^{\circ} \mathrm{C}$ and smoothly shaken for $1 \mathrm{~h}$ for adequate labeling, modifying the original protocol described by Makrigiorgos (1997). The probe $\mathrm{C}_{11}$-BODIPY581/591 is very sensitive to oxidation initiated by ROO and RO radicals (Drummen et al. 2002). The fluorescent probe $\mathrm{C}_{11}$-BODIPY581/591: (a) anchors with high stability to biological membranes by strong hydrophobic interaction of its undecanoic acid moiety with lipid bilayers; (b) shows good spectral separation of the non-oxidized (595 nm) and oxidized $(520 \mathrm{~nm}$ ) forms; (c) has a good photo-stability and displays very few fluorescence artifacts; (d) is virtually insensitive to microenvironmental changes, such as $\mathrm{pH}$ or solvent polarity; (e) once oxidized, $\mathrm{C}_{11}$-BODIPY581/591 remains lipophilic and does not spontaneously leave the lipid bilayer; (f) is not cytotoxic to most model mammal cells; and (g) is comparably sensitive to oxidation of common unsaturated fatty acids of biological membranes (Papa et al. 1999).

The progression of neutrophil membrane oxidation was measured by monitoring the decay of fluorescence emission at $600 \mathrm{~nm}$ for $60 \mathrm{~min}$ (excit. $\lambda=575 \mathrm{~nm}$ ), after the addition of $100 \mathrm{ng} /$ well phorbol-12-myristate-13-acetate (PMA), to trigger the oxidative burst in neutrophils. At time zero (immediately before PMA addition), fluorescence intensity was adjusted to 1.0 unit and, thus, hereafter defined as dimensionless relative fluorescence intensity (arbitrary units, A.U.). We assume that under these circumstances, the reaction of $\mathrm{ROO} / \mathrm{RO}$ radicals with $\mathrm{C}_{11^{-}}$ BODIPY581/591 becomes a pseudo first-order reaction, which is well fitted by the first-order exponential decay function:

$$
y=y_{0}+A_{1} e^{(-k t)} \text {. }
$$

where $y$ is relative fluorescence intensity, $y_{0}$ is relative fluorescence intensity at time zero (equals 1.0), $A_{1}$ is attenuation factor, $k$ is rate constant and $t$ is time (min).

\section{Statistical analyses}

Statistical analysis on $\mathrm{C}_{11}$-BODIPY581/591 kinetics was performed by paired student $t$ test of all 56 data points, followed by the Tukey's post-test. Origin Pro8 8SR0 software was used for statistical analyses and graph preparation (v8.0725/B725; OriginLab Corporation, Northampton, MA, USA). 


\section{Results}

Figure 1 illustrates the experimental controls of $\mathrm{C}_{11}$-BODIPY581/591 incorporated in neutrophil membranes. As shown in Fig. 1a, different AST concentration did not affect the (relatively noisy) fluorescent signal of $\mathrm{C}_{11}$-BODIPY581/591 pre-attached to membranes of non-activated neutrophils $\left(2 \times 10^{6}\right.$ cell/well). Similar kinetics were obtained with lower density cultures. Figure $1 \mathrm{~b}$ shows the unexpected increase of $\mathrm{C}_{11}$-BODIPY $581 / 591$ fluorescence in $2 \times 10^{6} \mathrm{cell} /$ well cultures of neutrophils activated with PMA. Interestingly, AST did not show any significant effect on $\mathrm{C}_{11}$ BODIPY581/591 kinetics. When cell density was diminished to $1 \times 10^{6}$ cell/well, $\mathrm{C}_{11^{-}}$ BODIPY ${ }^{51 / 591}$ fluorescence was apparently more responsive to AST concentrations, although fluorescence increases were still observed (Fig. 1c). Finally, increasing concentrations of AST proportionally reduced the fluorescence decay rate of $\mathrm{C}_{11}$ BODIPY581/591 in $5 \times 10^{5}$ neutrophil/well cultures, as shown in Fig. 1d. It is worthy to note that a transition period of about 9 min (ca. $500 \mathrm{~s}$ ) was necessary to obtain a stable fluorescence signal. Based on these results, further studies-as those under testosterone effect-were performed with $1 \times 10^{5}$ neutrophil/well cultures, which accordingly provided more stable fluorescent signals (Figs. 2, 3).

Based on calculated correlation indexes $\left(R^{2}>0.74\right.$; Table 1), the first-order exponential decay function adequately fits the fluorescence variation of $\mathrm{C}_{11}$ BODIPY $581 / 591$ with time, as a marker of neutrophil membrane oxidation. In the absence of PMA (non-activated neutrophils), a discrete progression of membrane oxidation was observed in control cells (open circles in Fig. 2), which suggests that the selected neutrophil isolation protocol adequately kept neutrophils in a nonactivated (rested) form. Quantitatively, the kinetic parameter $k$ is useful to estimate the rate of lipid oxidation in neutrophil membranes (Table 1). Figure 2 shows that 0.1 and $10 \mu \mathrm{M}$ TES significantly accelerated basal membrane oxidation of human neutrophils, albeit without PMA (chemical) activation. This effect is apparently dose-independent, although further studies are necessary to confirm this hypothesis.

As expected, PMA addition to control neutrophils significantly activated the oxidative burst, as shown by comparing open circles in Figs. 2 and 3, and based on the calculated $k$ values in Table 1 ( $k$ was 1.86-fold lower in the presence of PMA than in control neutrophils). On the other hand, PMA activation surprisingly diminished the degree of membrane oxidation in $0.1 \mu \mathrm{M}$ TES-treated neutrophils compared to non-activated cells ( $k$ was 2.74 -fold higher in PMA-activated neutrophils than in basal conditions), to reach identical scores as control neutrophils (Table 1). Contrarily, PMA addition did not alter the high rate of oxidation $(k)$ in membranes of human neutrophils pre-treated with $10 \mu \mathrm{M}$ TES for $24 \mathrm{~h}$. 

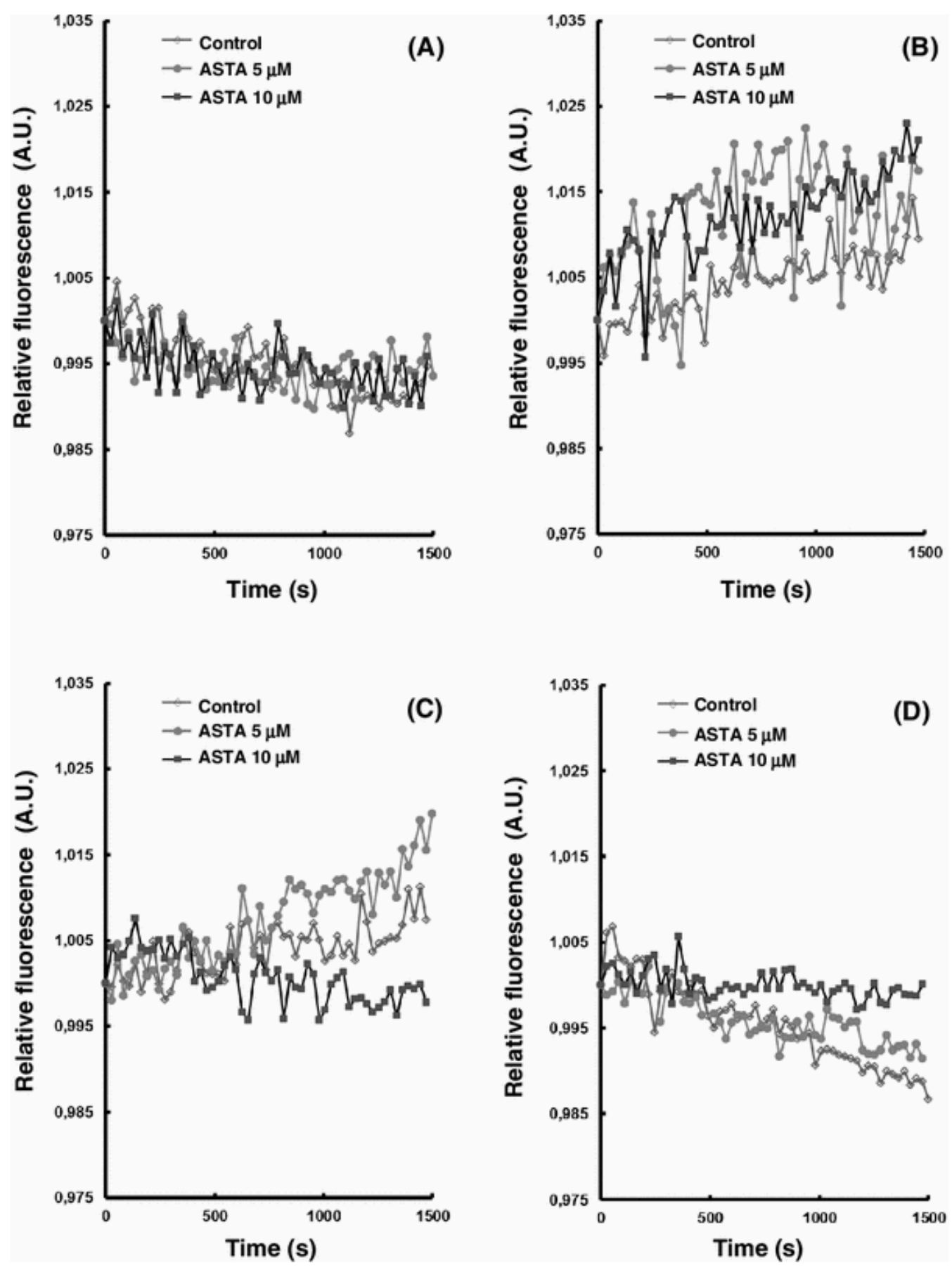

Fig. 1

Experimental controls for $\mathrm{C}_{11}$-BODIPY ${ }^{581 / 591}$ oxidation in human neutrophils. a Baseline fluorescence of for $\mathrm{C}_{11}$-BODIPY581/591 in $2 \times 10^{6}$ neutrophil/well cultures (non-activated) in the presence or absence of 5 and $10 \mu \mathrm{M}$ astaxanthin (ASTA); b Fluorescence kinetics of $\mathrm{C}_{11}$-BODIPY581/591 in $2 \times 10^{6}$ neutrophil/well cultures activated at time zero with $100 \mathrm{ng}$ PMA/well in the presence or absence of 5 and $10 \mu \mathrm{M}$ astaxanthin (ASTA); c Fluorescence kinetics of 
C11-BODIPY $^{581 / 591}$ in $1 \times 10^{6}$ neutrophil/well cultures activated at time zero with $100 \mathrm{ng}$ PMA/well in the presence or absence of 5 and $10 \mu \mathrm{M}$ astaxanthin (ASTA); and $\mathbf{d F l u o r e s c e n c e ~ k i n e t i c s ~ o f ~} \mathrm{C}_{11}$-BODIPY581/591 in $5 \times 10^{5}$ neutrophil/well cultures activated at time zero with $100 \mathrm{ng}$ PMA/well in the presence or absence of 5 and $10 \mu \mathrm{M}$ astaxanthin (ASTA)

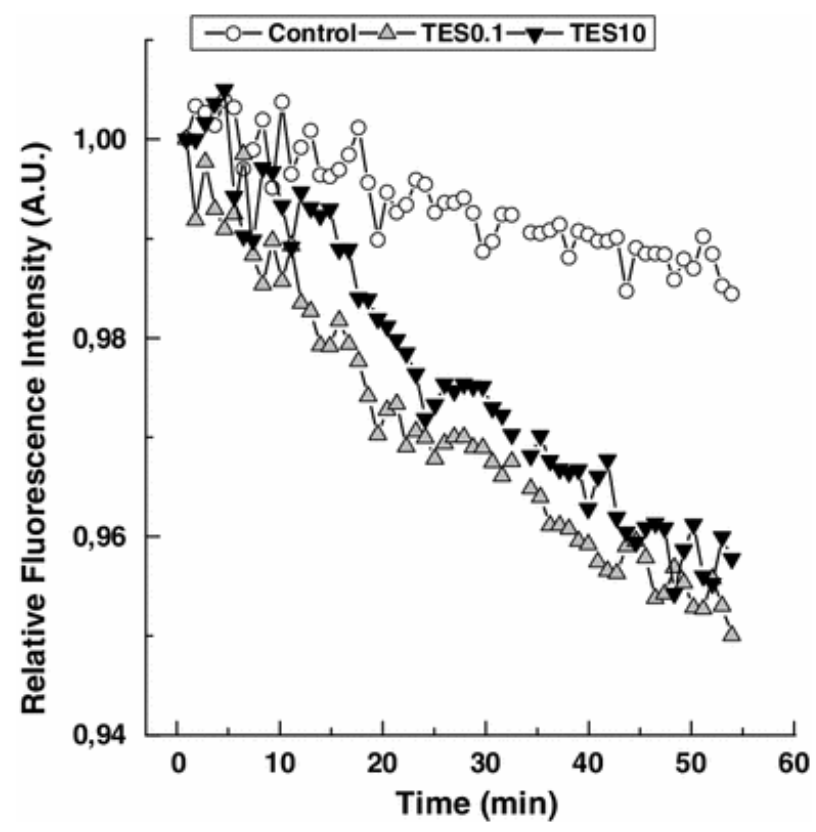

Fig. 2

Kinetics of $\mathrm{C}_{11}$-BODIPY581/591 fluorescence in human neutrophils treated for $24 \mathrm{~h}$ with 0.1 or $10 \mu \mathrm{M}$ testosterone (TES) in vitro

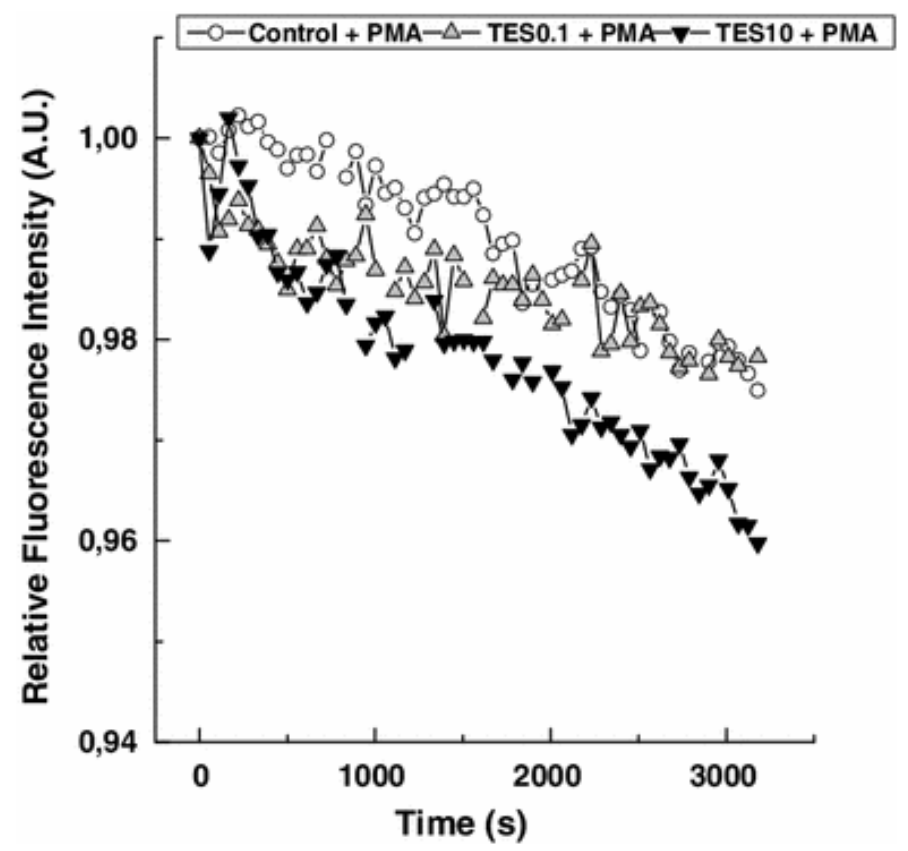




\section{Fig. 3}

Kinetics of $\mathrm{C}_{11}$-BODIPY581/591 fluorescence in human neutrophils treated for $24 \mathrm{~h}$ with 0.1 or $10 \mu \mathrm{M}$ testosterone (TES) in vitro and activated at time zero with $100 \mathrm{ng} /$ well phorbol-12-myristate-13-acetate (PMA)

\section{Table 1}

First-order decay parameters of $\mathrm{C}_{11}$-BODIPY581/591 fluorescence in resting and chemically activated ( $100 \mathrm{ng}$ PMA $/ 10^{5}$ cells) human neutrophils pre-treated in vitro with testosterone (TES) 0.1 or $10 \mu \mathrm{M}$ for $24 \mathrm{~h}$

\begin{tabular}{|l|l|l|l|l|}
\hline Groups & $Y_{0}$ & $A_{1}$ & $k\left(\times 10^{3}\right)$ & $R^{2}$ \\
\hline Controla,b,c & $0.910 \pm 0.275$ & $0.090 \pm 0.027$ & $18.45 \pm 0.26$ & 0.799 \\
\hline Control + PMA $, a, g, h$ & $0.911 \pm 0.301$ & $0.058 \pm 0.003$ & $9.90 \pm 2.63$ & 0.917 \\
\hline TES $0.1 \mu \mathrm{M}^{\mathrm{b}, \mathrm{d}, \mathrm{e}}$ & $0.935 \pm 0.005$ & $0.065 \pm 0.005$ & $2.49 \pm 0.37$ & 0.969 \\
\hline TES $0.1 \mu \mathrm{M}+\mathrm{PMA}, \mathrm{g}, \mathrm{i}$ & $0.910 \pm 0.046$ & $0.087 \pm 0.045$ & $6.82 \pm 0.46$ & 0.927 \\
\hline TES $10 \mu \mathrm{Mc}^{\mathrm{c}, \mathrm{e}, \mathrm{f}}$ & $0.911 \pm 0.019$ & $0.094 \pm 0.018$ & $4.31 \pm 1.26$ & 0.962 \\
\hline TES $10 \mu \mathrm{M}+\mathrm{PMA}$ f,h,i & $0.964 \pm 0.019$ & $0.030 \pm 0.019$ & $4.45 \pm 0.41$ & 0.738 \\
\hline
\end{tabular}

$A_{1}$ attenuation factor, $k$ exponential rate constant, PMA phorbol-12-myristate-13-acetate, TES testosterone, $y_{0}$ relative fluorescence intensity at time zero (initial data point $=1.0$ )

* Due to fluorescence oscillation, the kinetic parameters presented here were calculated excluding data points of the initial 2 min

Significantly different groups at:

$$
\begin{aligned}
& { }^{\mathrm{a}} p=1.1 \times 10^{-6} \\
& { }^{\mathrm{b}} p=7.5 \times 10^{-25} \\
& { }^{\mathrm{c}} p=1.2 \times 10^{-18} \\
& { }^{\mathrm{d}} p=5.6 \times 10^{-14} \\
& { }^{\mathrm{e}} p=2.3 \times 10^{-15} \\
& { }^{\mathrm{f}} p=2.3 \times 10^{-7} \\
& { }^{9} p=6.1 \times 10^{-30} \\
& { }^{\mathrm{h}} p=4.6 \times 10^{-11} \\
& { }^{\mathrm{i}} p=1.3 \times 10^{-11}
\end{aligned}
$$




\section{Discussion}

\section{Experimental procedures}

Independent to which protocol for neutrophil isolation was used (including pretreatment with $6 \%$ Dextran or not), noisy signals of fluorescence were detected during the first 5-10 min after PMA activation of the oxidative burst (Figs. 1, 2, 3). This effect was observed either when neutrophils were pre-loaded with the powerful antioxidant AST or by TES addition, which suggests that structural changes in cell membrane following PMA activation are the most probable molecular event involved. It is also plausible that the observed structural changes were caused by the organic solvent DMSO, which solubilizes the activation factor PMA. Nevertheless, after the stabilization period, the fluorescence signal of $\mathrm{C}_{11^{-}}$ BODIPY581/591 was only stable and apparently responsive to free radicals when neutrophil density was kept $<1 \times 10^{6}$ cell/well (Fig. 1a-d). Undesirable iron contamination in samples (probably from hemolysis) should also be kept in mind when evaluating quantitative parameters of membrane oxidation of neutrophils and other immune cells (Haag-Weber et al. 1989).

Unexpectedly, Fig. 1b, c (experiments performed neutrophil densities $>1 \times 10^{6}$ cell/well) revealed that $\mathrm{C}_{11}$-BODIPY581/591 fluorescence did not adequately respond to AST incorporation in neutrophil membranes. These results clearly demonstrate that the free radical-sensitive probe is not stoichiometrically exposed to ROS/RNS produced by active neutrophils. Physicochemical changes in neutrophil membranes due to cell-cell interactions (especially here, in high cell density cultures) might explain the unexpected pattern of fluorescence kinetics observed in Fig. 1b, c. Fluorescent probes, including $\mathrm{C}_{11}$-BODIPY581/591, normally change their excitation and emission spectra when in aggregate forms or upon strong cell-cell interactions, changes in membrane fluidity, loss of lipid raft integrity, and/or mechanical stress (de la Haba et al. 2012; Verstraeten et al. 2010). Nevertheless, by limiting cell-cell interactions in $5 \times 10^{5}$ cell/well cultures, $\mathrm{C}_{11^{-}}$ BODIPY581/591 (at a final concentration of $3.2 \mu \mathrm{M}$ ) was properly challenged by ROS/RNS generated by activated neutrophils, and the dose-dependent effect of AST (a powerful antioxidant) was adequately evidenced (Fig. 1d). Based on these results, further studies - as those with TES-were performed with $1 \times 10^{5}$ neutrophil/well cultures, which accordingly provided more stable fluorescent signals (Figs. 2, 3). As shown for other cell types, the novel approach presented here (the real-time monitoring of membrane oxidation in human neutrophils by $\mathrm{C}_{11}$-BODIPY581/591) seems to adequately reproduce the oxidative challenges faced by activated neutrophils, since PMA addition accelerated membrane oxidation (and permeability, Shasby et al. 1983) in control neutrophils due to ROS/RNS production. However, other chemical stimuli, e.g., granulocyte-macrophage colony-stimulating factor (GMCSF), lipopolysaccharide (LPS) or LPS in combination with interferon-gamma (IFN$\gamma)$, can also activate the oxidative burst in neutrophils through different signaling pathways (Atzeni et al. 2002). Therefore, further studies evaluating specific 
receptor-mediated activation of neutrophils are required, since PMA is a nonspecific polymorphonuclear cell activator (Paik et al. 2005).

\section{Effects of TES on membrane oxidation}

Testosterone treatment in vitro increased baseline membrane oxidation of nonactivated neutrophils in a dose-independent matter, as shown in Fig. 1 and ( $k$ values) in Table 1. However, we cannot confirm if this effect was caused by baseline overproduction of ROS/RNS in resting neutrophils and/or by TESmediated increase of antioxidant defenses. Marin et al. (2010) elegantly reported that pre-incubation with $10 \mathrm{nM}, 0.1$ and $10 \mu \mathrm{M}$ TES clearly inhibited superoxide radical $\left(\mathrm{O}_{2}{ }^{--}\right)$production in of human neutrophils after PMA activation (albeit, at a fivefold lower concentration). Under these circumstances, the authors reported lower indexes of lipid oxidation (using the thiobarbituric-acid reactive substances assay; TBARS) in $10 \mathrm{nM}$ and $0.1 \mu \mathrm{M}$ TES-treated cells, but not after $10 \mu \mathrm{M}$ TES incubation. Although the TBARS assay is less sensitive than the $\mathrm{C}_{11^{-}}$ BODIPY581/591 method applied here (Aldini et al. 2001), these data sustain the hypothesis that $0.1 \mu \mathrm{M}$ TES mediates suppressive effects on ROS production in PMAactivated neutrophils. Accordingly, Juliet et al. (2004) have already reported the suppressive effects of $0.01 \mu \mathrm{M}$ TES treatment (tenfold lower concentration than in our study) on NADPH oxidase assemblage (both p67 phox and p47phox expression). Regarding cellular mechanisms, Chignalia et al. (2012) demonstrated that $0.1 \mu \mathrm{M}$ TES induces vascular smooth muscle cells (VSMCs) migration via NADPH oxidasederived ROS and c-Src (a regulator of redox-sensitive migration) dependent pathways in hypertensive animals. The observed effect was promoted by both genomic and non-genomic mechanisms: TES increased Nox1 and Nox4 mRNA levels and $\mathrm{p} 47_{\text {phox }}$ protein expression, which are differentially regulated in VSMCs from Wistar-Kyoto rats and hypertensive rats (Chignalia et al. 2012). Thus, depending on the supra-physiological doses of TES, specific mechanisms are apparently activated to influence redox metabolism in neutrophils. Strong evidence points towards the assembling process and/or migration of NADPH oxidase sub-particles to neutrophil membrane (Juliet et al.2004; Chignalia et al. 2012).

Based on a well-accepted hormesis principle, too low ROS/RNS production would limit crucial redox-dependent processes such as cell signaling, $\mathrm{H}_{2} \mathrm{O}_{2}$-mediated proliferation, and oxidative burst responses in polymorphonuclear cells, whereas excessive ROS/RNS concentrations would cause undesirable oxidative modifications to important cellular biomolecules (Valko et al. 2007). Other authors have also reported dose-dependent ambiguous effects of TES in immune cells, at the same micromolar range (Greabu et al. 2003; Juliet et al. 2004). Although TES concentrations reported here represent $10^{2}$ to $10^{4}$-fold higher doses than those physiologically found in average men (Shehzad et al. 2001), our study demonstrates that supra-physiological doses of TES can distinctively affect the progression of membrane oxidation in activated neutrophils, a molecular event that is truthfully related to cell viability, neutrophil turnover scores, and immune responsiveness. 


\section{Conclusions}

Altogether, our results show that supra-physiological doses of TES impose higher oxidative conditions on circulating (non-activated) neutrophils. This may enhance the general antioxidant capacity of circulating neutrophils as a response to the TESinduced baseline oxidation of membranes. On the other hand, $0.1 \mu \mathrm{M}$ TES treatment appropriately restored membrane oxidation rates back to those found in PMAactivated control neutrophils, whereas the pre-treatment with $10 \mu \mathrm{M}$ TES (100-fold higher concentration) sustained the high susceptibility of neutrophils to autoinduced membrane oxidation. Thus, the tricky aspect of obtaining maximal benefits from medical TES treatments (e.g., hormone reposition, post-surgery recovery, etc.) is to apply an optimized TES concentration-matching its "pharmacological window"-that appropriately balances its required anabolic and/or androgenic effects with related immunosuppressive effects due to increased membrane oxidation in activated neutrophils. In a much worse scenario involving circulating neutrophils, it is worthwhile mentioning that TES benefits are normally obtained at nano/micro molar concentrations in plasma, which, thus, require sensitive methods to investigate such relevant physiological processes. The technique presented here represents a useful tool to investigate that hypothesis.

\section{Acknowledgments}

The authors are indebted to Dr. Rosemari Otton, and M.Sc. Douglas Marin Popp for providing neutrophil samples and resources during preliminary studies at Laboratório de Fisiologia Celular, campus Analia Franco, Universidade Cruzeiro do Sul, São Paulo, Brazil, and the assistance of Dr. Douglas Ganini for neutrophil isolation in further experiments. Dr. Marcelo Paes de Barros is also indebted to Prof. Rui Curi, at Department of Biophysics and Physiology, University of São Paulo, Brazil, for chemicals and laboratory facilities during neutrophil isolation. This research is supported by Fundação de Amparo a Pesquisa do Estado de Sao Paulo (FAPESP 2009/12342-8), Programa de Suporte à Pós-graduação de Instituições de Ensino Particulares, da Coordenação de Aperfeiçoamento de Pessoal de Nível Superior (PROSUP/CAPES, Brazil), and Conselho Nacional de Desenvolvimento Científico e Tecnológico (Bolsas Produtividade em Pesquisa, Nivel 2, \#312404/2009-3, CNPq, Brazil).

\section{References}

Aldini G, Yeum KJ, Russell RM, Krinsky NI (2001) A method to measure the oxidizability of both the aqueous and lipid compartments of plasma. Free Radic Biol Med 31:1043-1050 
Atzeni F, Schena M, Ongari M, Carrabba M, Bonara P, Minonzio F, Capsoni F (2002) Induction of CD69 activation and molecule on human neutrophils by GM-CFS, IFNgamma, and IFN-alpha. Cell Immunol 220:20-29

Axell AM, MacLean HE, Plant DR, Harcourt LJ, Davis JA, Jimenez M, Handelsman DJ, Lynch GS, Zajac JD (2006) Continuous testosterone administration prevents skeletal muscle atrophy and enhances resistance to fatigue in orchidectomized male mice. Am J Physiol Endocrinol Metab 291:E506-E516

Barros MP, Pinto E, Colepicolo P, Pedersén M (2001) Astaxanthin and peridinin inhibit oxidative damage in $\mathrm{Fe}(2+)$-loaded liposomes: scavenging oxyradicals or changing membrane permeability? Biochem Biophys Res Commun 288:225-232

Chignalia AZ, Schuldt EZ, Camargo LL, Montezano AC, Callera GE, Laurindo FR, Lopes LR, Avellar MCW, Carvalho MHC, Fortes ZB, Touyz RM, Tostes RC (2012)

Testosterone induces vascular smooth muscle cell migration by NADPH oxidase and c-Src-dependent pathways. Hypertension 59:1263-1271

de la Haba C, Palacio JR, Martínez P, Morros A (2012) Effect of oxidative stress on plasma membrane fluidity of THP-1 induced macrophages. Biochim Biophys Acta (BBA)-Biomembranes.

Dillon EL, Durham WJ, Urban RJ, Sheffield-Moore M (2010) Hormone treatment and muscle anabolism during aging: androgens. Clin Nutr 29:697-700

Dong J, Chen P, Wang R, Yu D, Zhang Y, Xiao W (2011) NADPH oxidase: a target for the modulation of the excessive oxidase damage induced by overtraining in rats neutrophils. Int J Biol Sci 7:881-891

Drummen GP, van Liebergen LC, Op den Kamp JA, Post JA (2002) C11-

BODIPY(581/591), an oxidation-sensitive fluorescent lipid peroxidation probe: (micro)spectroscopic characterization and validation of methodology. Free Radic Biol Med 33:473-490 
Freitas M, Porto J, Lima JL, Fernandes E (2008) Isolation and activation of human neutrophils in vitro. The importance of the anticoagulant used during blood collection. Clin Biochem 41:570-575

Greabu M, Olinescu R, Totan A (2003) Possible regulatory effects of steroid hormones on the chemiluminescence produced by activated polymorphouclear leukocytes. Acta Medica Bulgarica 30:36-45

Haag-Weber M, Schollmeyer P, Hörl WH (1989) Neutrophil activation during hemodialysis. Adv Exp Med Biol 260:27-37

Hoffman JR, Kraemer WJ, Bhasin S, Storer T, Ratamess NA, Haff GG, Willoughby DS, Rogol AD (2009) Position stand and androgen and human growth hormone use. J Strength Cond Res 23:S1-S59

Juliet PA, Hayashi T, Daigo S, Matsui-Hirai H, Miyazaki A, Fukatsu A, Funami J, Iguchi A, Ignarro LJ (2004) Combined effect of testosterone and apocynin on nitric oxide and superoxide production in PMA-differentiated THP-1 cells. Biochim Biophys Acta 1693:181-191

Krüeger K, Frost S, Most E, Volker K, Pallauf J, Mooren FC (2009) Exercise affects tissue lymphocyte apoptosis via redox-sensitive and Fas-dependent signaling pathways. Am J Physiol Regul Integr Comp Physiol 296:R1518-R1527

Liva SM, Voskuhl RR (2001) Testosterone acts directly on CD4 + T lymphocytes to increase IL-10 production. J Immunol 167:2060-2067

Makrigiorgos GM (1997) Detection of lipid peroxidation on erythrocytes using the excimer-forming property of a lipophilic BODIPY fluorescent dye. J Biochem Biophys Methods 35:23-35 
Malkin CJ, Pugh PJ, Jones RD, Kapoor D, Channer KS, Jones TH (2004) The effect of testosterone replacement on endogenous inflammatory cytokines and lipid profiles in hypogonadal men. J Clin Endocrinol Metab 89:3313-3318

Marin DP, Bolin AP, Dos de C Santos R, Curi R, Otton R (2010) Testosterone supresses oxidative stress in human neutrophils. Cell Biochem Funct 28:394-402

Naguib YMA (2000) Antioxidant activities of astaxanthin and related carotenoids. J Agric Food Chem 48:1150-1154

Ottonello L, Frumento G, Arduino N, Dapino P, Tortolina G, Dallegri F (2011) Immune complex stimulation of neutrophil apoptosis: investigating the involvement of oxidative and nonoxidative pathways. Free Radic Biol Med 30:161-169

Paik JY, Ko BH, Choe YS, Choi Y, Lee KH, Kim BT (2005) PMA-enhanced neutrophil [18F]FDG uptake is independent of integrin occupancy but requires PI3 K activity. Nucl Med Biol 32:561-566

Papa EHW, Drummen GPC, Winter VJ, Kooij TWA, Rijken P, Wirtz KWA, Op den Kamp JAF, Hage WJ, Post JA (1999) Ratio-Euorescence microscopy of lipid oxidation in living cells using C11-BODIPY581/591. FEBS Lett 453:278-282

Shasby DM, Shasby SS, Peach MJ (1983) Granulocytes and phorbol myristate acetate increase permeability to albumin of cultured endothelial monolayers and isolated perfused lungs. Role of oxygen radicals and granulocyte adherence. Am Rev Resp Dis 127:72-76

Shehzad B, Wahlstrom JT, Dobs AS (2001) Anabolic-androgenic steroid therapy in the treatment of chronic diseases. J Clin Endocrinol Metab 86:5108-5117

Thompson RW, McClung JM, Baltgalvis KA, Davis JM, Carson JA (2006) Modulation of overload-induced inflammation by aging and anabolic steroid administration. Exp Gerontol 41:1136-1148 
Valko M, Leibfritz D, Moncol J, Cronin MT, Mazur M, Telser J (2007) Free radicals and antioxidants in normal physiological functions and human disease. Int J Biochem Cell Biol 39:44-84

Verstraeten SV, Mackenzie GG, Oteiza PI (2010) The plasma membrane plays a central role in cells response to mechanical stress. Biochim Biophys Acta (BBA) -. Biomembranes 1798:1739-1749

Zembron-Lacny A, Slowinska-Lisowska M, Ziemba A (2010) Integration of the tiol redox status with cytokine response to physical training in professional basketball players. Physiol Res 59:239-245 